\title{
Screening of key genes associated with R-CHOP immunochemotherapy and construction of a prognostic risk model in diffuse large B-cell lymphoma
}

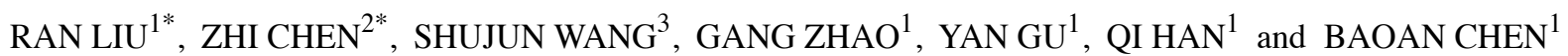 \\ ${ }^{1}$ Department of Hematology and Oncology, Zhongda Hospital Southeast University; ${ }^{2}$ Department of Cardiology, \\ Jiangsu Province Hospital; ${ }^{3}$ Department of Blood Transfusion, Nanjing General Hospital of PLA, \\ Nanjing, Jiangsu 210009, P.R. China
}

Received February 18, 2019; Accepted July 25, 2019

DOI: $10.3892 / \mathrm{mmr} .2019 .10627$

\begin{abstract}
Diffuse large B-cell lymphoma (DLBCL) is a common subtype of non-Hodgkin lymphoma, which is curable in the majority of patients treated with rituximab plus cyclophosphamide, doxorubicin, vincristine and prednisone (R-CHOP) immunochemotherapy. However, the therapeutic mechanism of R-CHOP has not been elucidated. The GSE32918 and GSE57611 datasets were retrieved from The Gene Expression Omnibus database. The differentially expressed genes (DEGs) associated with R-CHOP therapy were identified using limma. Combined with prognostic information in GSE32918, DEGs found to be significantly associated with prognosis were selected using univariate Cox regression analysis and a risk prediction model was constructed. Based on this model, the samples in the training set (GSE32918) were divided into high and low risk score groups according to the median risk score. A total of 801 DEGs were identified between the R-CHOP treated DLBCL and primary DLBCL samples, from this 116 prognosis-associated genes were selected. Using Cox proportional hazards model, an optimal combination of 12 genes [including calcium/calmodulin dependent protein kinase I (CAMK1), hippocalcin like 4 (HPCAL4) and ephrin A5 (EFNA5)] was selected, and the sample risk score prediction model was constructed and validated. The DEGs between high risk score and low risk score groups were significantly
\end{abstract}

Correspondence to: Dr Ran Liu or Dr Baoan Chen, Department of Hematology and Oncology, Zhongda Hospital Southeast University, Building 2, 87 Dingjiaqiao Road, Gulou, Nanjing, Jiangsu 210009, P.R. China

E-mail: liu_ran30@163.com

E-mail: cba8888@hotmail.com

*Contributed equally

Key words: diffuse large B-cell lymphoma, rituximab plus cyclophosphamide, doxorubicin, vincristine, and prednisone, differentially expressed genes, prognosis risk model enriched in functions associated with 'response to DNA damage stimulus', and pathways including 'cytokine-cytokine receptor interaction' and 'cell cycle'. The optimal combination of the 12 genes, including CAMK1, HPCAL4 and EFNA5, was found to be useful in predicting the prognosis of patients with DLBCL after R-CHOP treatment. Therefore, these genes may be affected by R-CHOP in DLBCL.

\section{Introduction}

Diffuse large B-cell lymphoma (DLBCL) is a B-cell malignancy. B-cells are a type of white blood cell responsible for producing antibodies. DLBCL is the most common subtype of non-Hodgkin lymphoma among adults, accounting for $25-40 \%$ of new cases annually worldwide (1). CD20, a cell-surface protein that is expressed almost exclusively on mature B-cells, is expressed in $>90 \%$ of DLBCL cases (2). The conventional chemotherapy regimen for DLBCL has been dominated by cyclophosphamide, doxorubicin, vincristine and prednisone (CHOP) since the 1970s. Patients with advanced stage DLBCL have a remission rate of $45-55 \%$ and a cure rate of $30-35 \%$ with standard CHOP chemotherapy (3). However, it is known that drug resistance is a major obstacle to the success of cancer chemotherapy (4). For DLBCL, $60-70 \%$ of patients relapse or develop resistance to CHOP chemotherapy, with a 5-year overall survival rate of 40-50\% (5).

Rituximab is a chimeric IgG1 monoclonal antibody targeting CD20. The CD20-binding region of rituximab was derived from a mouse monoclonal antibody through genetic engineering $(4,6)$. It has been reported that rituximab is effective for the treatment of refractory or relapsed lymphomas, and that it also has activity in refractory or relapsed DLBCL $(7,8)$. Since the late 1990s, adding rituximab to conventional CHOP (R-CHOP) for DLBCL treatment has significantly improved the survival rate across all risk groups $(6,9)$. In 2003, R-CHOP was proposed as a first-line standard therapy for DLBCL in the National Comprehensive Cancer Network guideline $(10,11)$. DLBCL is curable in the majority of patients treated with R-CHOP (12), nevertheless, the therapeutic mechanism of $\mathrm{R}-\mathrm{CHOP}$ has not been elucidated. 
In the present study, the genes associated with R-CHOP therapy were selected by comparing expression in primary DLBCL with DLBCL after the treatment with R-CHOP. Additionally, a prognosis prediction model for patients with DLBCL treated with R-CHOP was constructed based on prognosis-related genes. The present study comprehensively screened for genes associated with R-CHOP therapy in DLBCL, and evaluated the relationship between gene expression and prognosis.

\section{Materials and methods}

Data screening and normalization. Expression profiles were retrieved from the National Center of Biotechnology Information Gene Expression Omnibus (ncbi.nlm.nih. gov/geo/) database with 'diffuse large B-cell lymphoma', 'Homo sapiens' and 'R-CHOP' as the key words. The selection criteria for the dataset were as follows: i) Data are gene expression profiles; ii) data from solid tumors at lymph nodes samples from patients with DLBCL (not blood or cell lines); iii) datasets contained DLBCL samples that did not receive and received R-CHOP treatment; and iv) data also had clinical prognostic information.

In total, two datasets (GSE32918 and GSE57611) $(13,14)$ were included in the present study. The GSE32918 dataset [including 82 germinal center B-cell like (GCB), 53 activated $\mathrm{B}$-cell $(\mathrm{ABC})$ and 37 Type III DLBCL] was derived from the GPL14951 Illumina HumanHT-12 WG-DASL V4.0 R2 expression beadchip platform (Illumina, Inc.). In the GSE32918 dataset there are 249 samples from formalin-fixed, paraffin-embedded biopsies (including $53 \mathrm{ABC}, 82 \mathrm{GCB}$ and 37 type III), including 62 DLBCL samples from 32 patients not treated with R-CHOP (palliative care or local radiotherapy) and 187 DLBCL samples from 140 patients treated with R-CHOP. Prognostic information was available for 167 patients. This dataset was used as the training dataset. The GSE57611 dataset, derived from the GPL96 [HG-U133A] Affymetrix Human Genome U133A Array (Affymetrix; Thermo Fisher Scientific, Inc.), included 148 DLBCL samples (including 79 GCB, 49 ABC and 20 unclassified DLBCLs), among which 30 samples had prognostic information. This dataset was used for validation.

For the GSE32918 dataset that was sequenced on the Illumina platform, the R package limma (version 3.34.0; https://bioconductor.org/packages/release/bioc/html/limma. html) (15) was used to transform the skewness distribution of the data to an approximately normal distribution. The data were then normalized using the median normalization method (16). Additionally, for the GSE57611 dataset sequenced using the Affymetrix platform, the R package oligo (version 1.42.0; bioconductor.org/packages/release/bioc/html/oligo.html) (17) was used for format conversion, filling missing data (median method) (18), background correction and data normalization (quantile method) (19).

Selection of differentially expressed genes (DEGs). Limma version 3.34.0 (15) in $\mathrm{R} 3.4 .1$ was used to calculate the false discovery rate (FDR) and fold change (FC) value of genes between the primary DLBCL sample and R-CHOP treated DLBCL samples in the training dataset. The genes

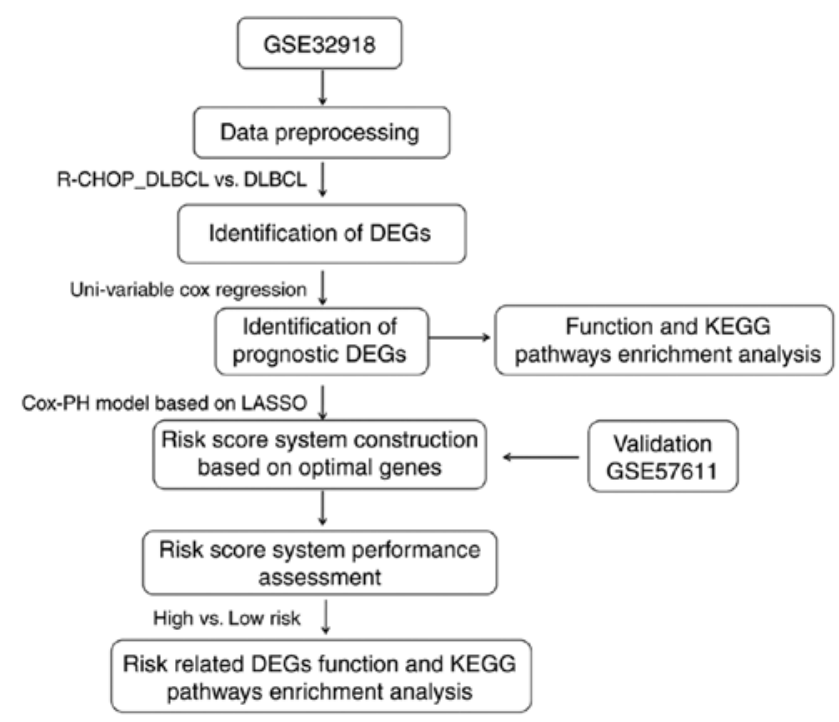

Figure 1. Analysis process. The GSE32918 dataset was used for the identification of DEGs. R-CHOP, rituximab plus cyclophosphamide, doxorubicin, vincristine, and prednisone immunochemotherapy; DLBCL, diffuse large B-cell lymphoma; DEGs, differentially expressed genes; KEGG, Kyoto Encyclopedia of Genes and Genomes; Cox-PH, Cox proportional hazards model.

with an $\mathrm{FDR}<0.05$ and $\mid \log \mathrm{FCl}>0.5$ were considered as DEGs. On the basis of the expression level of the DEGs, Euclidean distance (20) based bilateral hierarchical clustering (21) was performed using pheatmap (version 1.0.8; https://cran.r-r-project.org/package=pheatmap) (22) in R 3.4.1 and the results were visualized using a heatmap.

\section{Construction and validation of the prognostic risk prediction model}

Selection of the optimum combination of prognostic genes. The DEGs significantly associated with prognosis in the training set were further selected using the univariate Cox regression analysis in Survival (version 2.4; cran.r-project.org/web/packages/survival/index.html) (23) in R 3.4.1. A log-rank $\mathrm{P}<0.05$ was used as the threshold. The prognosis-associated DEGs were used for Gene Ontology (GO) function and Kyoto Encyclopedia of Genes and Genomes (KEGG) pathway enrichment analyses using the Database for Annotation, Visualization and Integrated Discovery (version 6.8; david. ncifcrf.gov/) (24). Subsequently, the optimum combination of prognostic genes were selected according to the hazard parameter ' $\lambda$ ' that was calculated 1,000 times using a cross-validation likelihood algorithm based on the Cox proportional hazards (Cox-PH) model (25) of L1-penalized regularization regression algorithm in the Penalized package version 0.9-51 (26) in R 3.4.1.

Establishment and verification of the risk prediction model. Based on the aforementioned DEGs combination and Cox-PH regression coefficients, a risk score system for each sample was established using gene expression values weighted by regression coefficients. The risk score of each sample was calculated as follows: Risk score $=\beta_{\text {gene } 1} \times$ expression $_{\text {gene } 1}+$

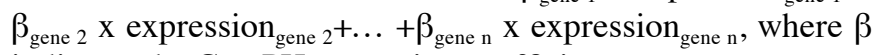
indicates the Cox-PH regression coefficient. 

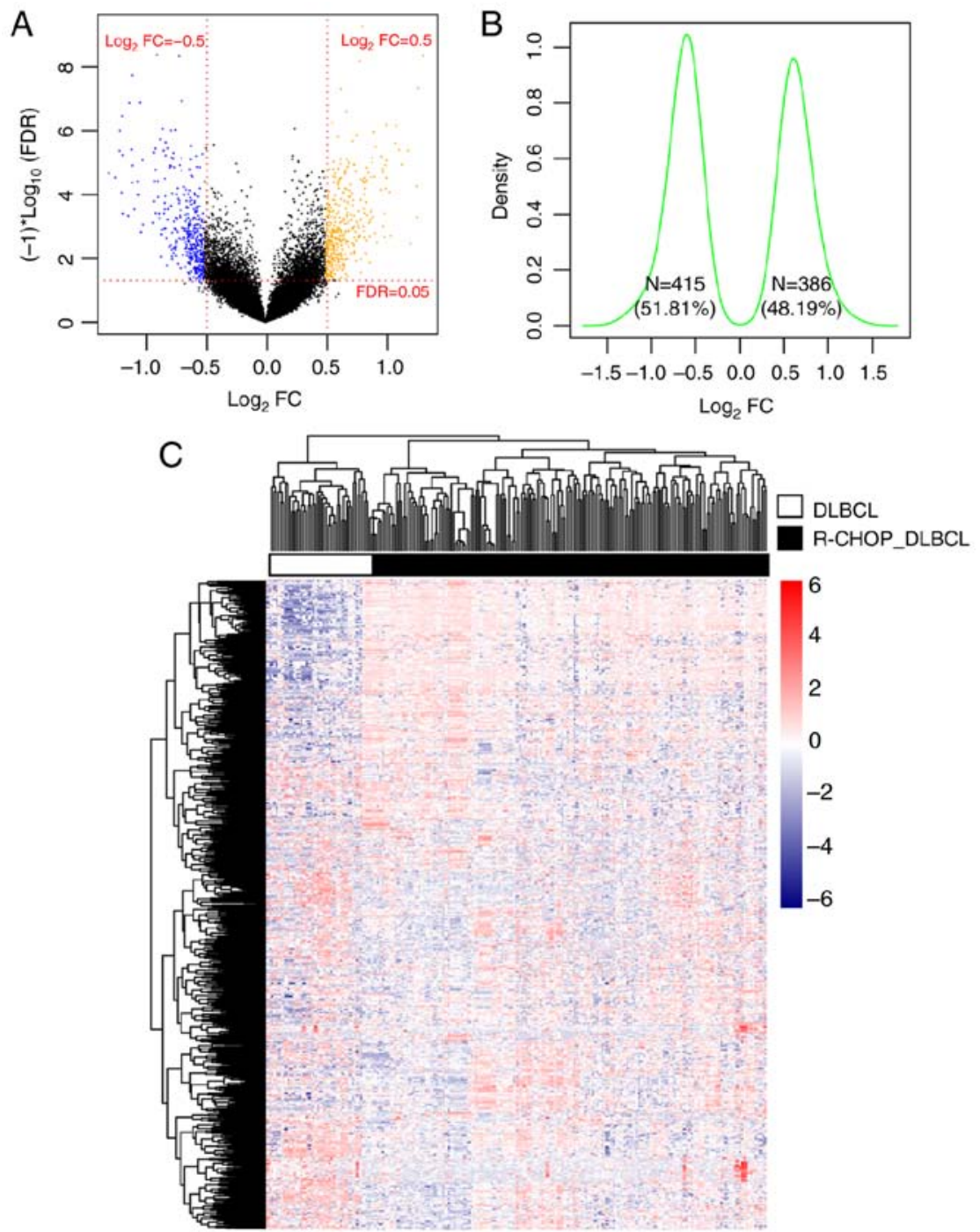

Figure 2. Results of screening for DEG. (A) Volcano plot. Orange and blue dots indicate significantly upregulated and downregulated DEGs, respectively. The red dotted horizontal line indicates an FDR $<0.05$ and the two red dotted vertical lines indicate $\mid \log F C l>0.5$. (B) $\log _{2}$ Kernel density curve based on DEGs. (C) Bidirectional hierarchical clustering heatmap based on the expression level of the DEGs. The heatmap colors indicate the expression level; blue indicates low expression while red indicates high expression. DEGs, differentially expressed genes; FDR, false discovery rate; FC, fold change; DLBCL, diffuse large B-cell lymphoma; R-CHOP, rituximab plus cyclophosphamide, doxorubicin, vincristine, and prednisone immunochemotherapy.

The samples in the training set were divided into high risk score and low risk score groups according to the median risk score. The correlation between the risk prediction model and prognosis was evaluated using Kaplan-Meier survival curves in Survival version 2.41-1 in R 3.4.1. The correlation was verified in the validation dataset (GSE57611).

Function analysis of DEGs between high and low risk score groups. The DEGs between high and low risk score groups were selected using limma version 3.34 .0 (15) with thresholds of $\mathrm{FDR}<0.05$ and $\log _{2} \mathrm{FCl}>0$. DEGs were considered to be significantly correlated with risk grouping, and were then subjected to GO and KEGG enrichment analyses, if the threshold of $\mathrm{P}<0.05$. The analysis process is shown in Fig. 1.

\section{Results}

Data preprocessing and DEG screening. After normalization, the DEGs between R-CHOP-treated and primary DLBCL samples were screened. In total, 801 DEGs were identified, including 386 that were upregulated and 415 that were downregulated (Fig. 2A). The $\log _{2}$ Kernel density curve based on the expression of the DEGs is shown in Fig. 2B. As shown in Fig. 2B, 51.81\% (415/801) of DEGs were significantly downregulated and $48.19 \%$ (386/801) of DEGs were significantly upregulated in R-CHOP treated DLBCL samples. The bidirectional hierarchical clustering heatmap based on the expression levels of the DEGs is shown in Fig. 2C. The DEGs in the different samples separated clearly, as represented by the distinct colors in the heatmap.

Construction and validation of the prognostic risk prediction model

Selection of the optimal combination of prognostic genes. Based on the identified DEGs and the prognostic information in GSE32918, a total of 116 genes that were significantly correlated with prognosis were selected using univariate Cox regression analysis. Functional analysis of the 116 genes 
Table I. GO and KEGG pathway enrichment results for the differentially expressed genes that were significantly correlated with prognosis.

A, Biological process

\begin{tabular}{lrr}
\hline Term & Count & P-value \\
\hline GO:0007411 axon guidance & 6 & 0.003641 \\
GO:0007229 integrin-mediated signaling pathway & 5 & 0.003814 \\
GO:0045892 negative regulation of transcription, DNA-templated & 10 & 0.004883 \\
GO:0045893 positive regulation of transcription, DNA-templated & 10 & 0.005975 \\
GO:0016055 Wnt signaling pathway & 6 & 0.007186 \\
GO:0007165 signal transduction & 5 & 0.00733 \\
GO:0051726 regulation of cell cycle & 5 & 0.008414 \\
GO:0030036 actin cytoskeleton organization & 9 & 0.009895 \\
GO:0043547 positive regulation of GTPase activity & 17 & 0.029303 \\
GO:0006355 regulation of transcription, DNA-templated & 5 & 0.030824 \\
GO:0042127 regulation of cell proliferation & 0.031547 \\
\hline
\end{tabular}

B, Cellular component

\begin{tabular}{lcc}
\hline Term & Count & P-value \\
\hline GO:0016020 membrane & 25 & 0.003065 \\
GO:0005667 transcription factor complex & 6 & 0.00656 \\
GO:0005886 plasma membrane & 36 & 0.018833 \\
\hline
\end{tabular}

C, Molecular function

\begin{tabular}{lrr}
\hline Term & Count & P-value \\
\hline GO:0005125 cytokine activity & 5 & 0.025713 \\
GO:0005515 protein binding & 67 & 0.027873 \\
GO:0003700 transcription factor activity, sequence-specific DNA binding & 12 & 0.041048 \\
GO:0044212 transcription regulatory region DNA binding & 5 & 0.04659 \\
\hline
\end{tabular}

D, KEGG pathway

\begin{tabular}{lcc}
\hline Term & Count & P-value \\
\hline hsa04060:Cytokine-cytokine receptor interaction & 7 & 0.00103 \\
hsa04014: Ras signaling pathway & 6 & 0.00354 \\
hsa04015:Rap1 signaling pathway & 5 & 0.008964 \\
hsa05200:Pathways in cancer & 7 & 0.009679 \\
hsa04062:Chemokine signaling pathway & 4 & 0.019075 \\
hsa04151:PI3K-Akt signaling pathway & 5 & 0.030569 \\
hsa04310:Wnt signaling pathway & 3 & 0.030827 \\
hsa04514:Cell adhesion molecules (CAMs) & 3 & 0.032028
\end{tabular}

GO, Gene Ontology; KEGG, Kyoto Encyclopedia of Genes and Genomes.

identified 18 significant GO terms, including 11 biological processes (BP), four molecular functions (MF) and three cellular components (CC), and eight KEGG pathways (Table I). The results of this analysis showed that the DEGs identified were significantly associated with the cell cycle and transcription regulation associated biological processes, as well as significantly involved in 'hsa04060:cytokine-cytokine receptor interaction' and 'hsa04014:Ras signaling pathway'. 

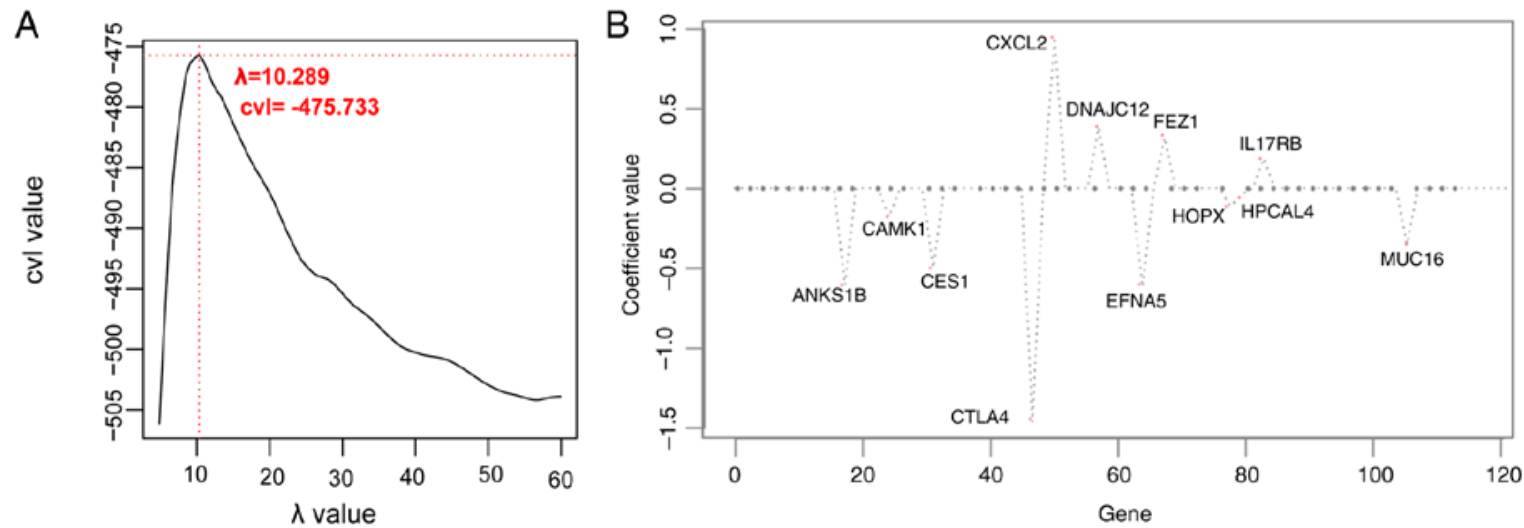

Figure 3. Selection of optimal prognostic genes. (A) Curve of cvl screening for the $\lambda$ parameter. The optimized parameter $\lambda$, indicator of the hazard, was obtained by 1,000 rounds of cross-validated likelihood (cvl) circular calculation. The horizontal and vertical axes represent different values of $\lambda$ and cvl, respectively. The crossing of the red dotted line represents $\lambda$ of 10.289 when cvl takes the maximum (-475.733). (B) Coefficient distribution diagram of the optimal combination of prognostic genes based on the Cox proportional hazards model. cvl, cross-validation likelihood; ANKS1B, ankyrin repeat and sterile $\alpha$ motif domain-containing protein 1B; CAMK1, calcium/calmodulin dependent protein kinase I; CES, carboxylesterase 1; CTLA4, cytotoxic T-lymphocyte associated protein 4; CXCL2, C-X-C motif chemokine ligand 2; DNAJC12, DnaJ heat shock protein family member C12; EFNA5, ephrin A5; FEZ1, fasciculation and elongation protein $\zeta 1$; HOPX, HOP homeobox; HPCAL4, hippocalcin like 4; IL17RB, interleukin 17 receptor B; MUC16, mucin 16, cell surface associated.

A

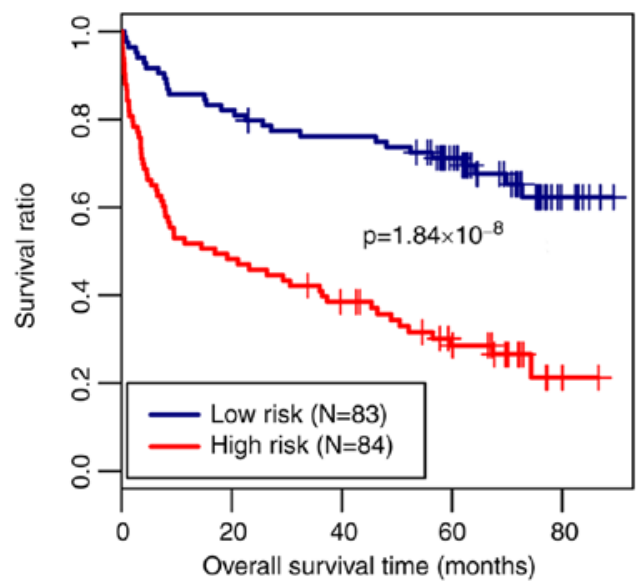

B

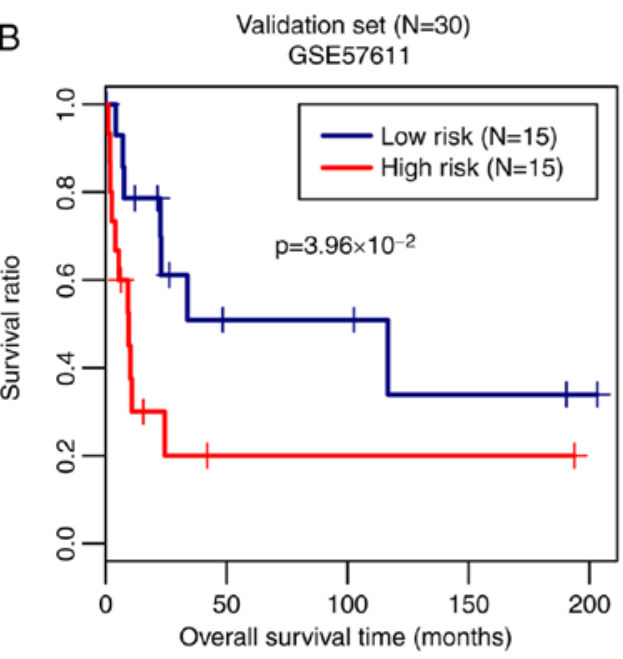

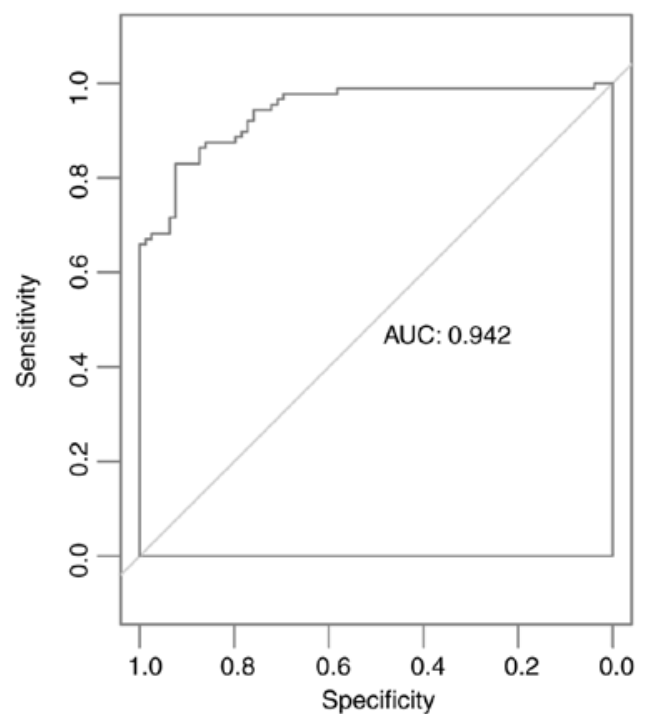

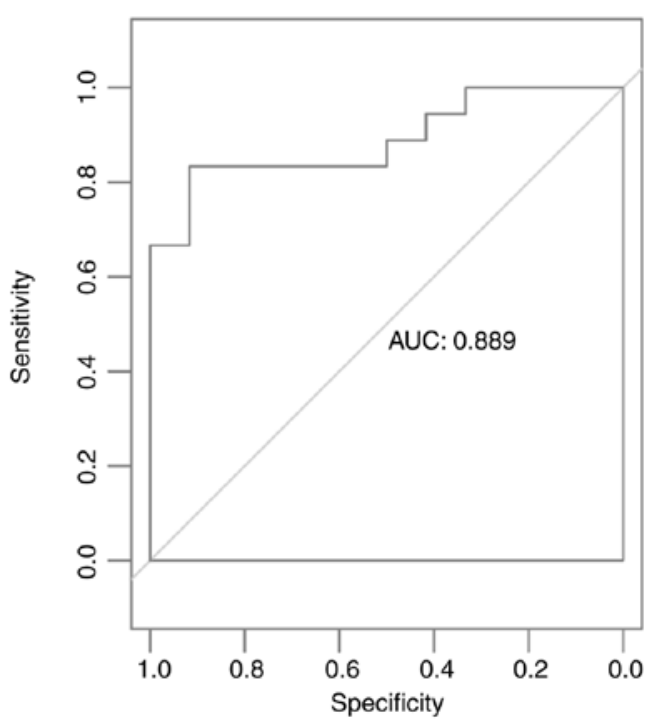

Figure 4. Prediction model and overall survival Kaplan-Meier curves. Kaplan-Meier overall survival curves (left) and receiver operating characteristic curves (right) based on the optimal gene combination in (A) training set (GSE32918) and (B) validation set (GSE57611). Blue and red curves represent the low and high risk score groups, respectively. AUC, area under curve. 
Table II. Optimal genes list based on Cox proportional hazards model of L1-penalized regularization regression algorithm.

\begin{tabular}{lrcc}
\hline Gene & Coef & Hazard ratio & P-value \\
\hline ANKS1B & -0.605 & $0.767(0.658-0.895)$ & $6.568 \times 10^{-4}$ \\
CAMK1 & -0.179 & $0.837(0.729-0.959)$ & $1.045 \times 10^{-2}$ \\
CES1 & -0.496 & $0.846(0.751-0.953)$ & $5.423 \times 10^{-3}$ \\
CTLA4 & -1.468 & $0.716(0.627-0.817)$ & $5.008 \times 10^{-7}$ \\
CXCL2 & 0.948 & $1.559(1.219-1.994)$ & $5.637 \times 10^{-4}$ \\
DNAJC12 & 0.379 & $1.480(1.239-1.767)$ & $1.332 \times 10^{-5}$ \\
EFNA5 & -0.619 & $0.811(0.719-0.915)$ & $5.862 \times 10^{-4}$ \\
FEZ1 & 0.317 & $1.214(1.059-1.390)$ & $5.066 \times 10^{-3}$ \\
HOPX & -0.114 & $0.859(0.750-0.984)$ & $2.703 \times 10^{-2}$ \\
HPCAL4 & -0.039 & $0.666(0.531-0.837)$ & $4.057 \times 10^{-4}$ \\
IL17RB & 0.187 & $1.137(1.023-1.263)$ & $1.627 \times 10^{-2}$ \\
MUC16 & -0.348 & $0.837(0.731-0.957)$ & $8.829 \times 10^{-3}$ \\
\hline
\end{tabular}

Coef, coefficient; ANKS1B, ankyrin repeat and sterile $\alpha$ motif domain-containing protein $1 \mathrm{~B}$; CAMK1, calcium/calmodulin dependent protein kinase I; CES, carboxylesterase 1; CTLA4, cytotoxic T-lymphocyte associated protein 4; CXCL2, C-X-C motif chemokine ligand 2; DNAJC12, DnaJ heat shock protein family member $\mathrm{C} 12$; EFNA5, ephrin A5; FEZ1, fasciculation and elongation protein $\zeta 1$; HOPX, HOP homeobox; HPCAL4, hippocalcin like 4; IL17RB, interleukin 17 receptor B; MUC16, mucin 16, cell surface associated.

The optimal combination of prognostic genes was determined using the Cox-PH model. When the parameter ' $\lambda$ ' was 10.289 , the cross-validation likelihood reached the maximum value of -475.733 (Fig. 3A). Under this parameter, the Cox-PH model identified a 12 gene combination (Fig. 3B). The 12 genes are listed in Table II.

Establishment and evaluation of the risk prediction model. According to the 12-gene combination, as well as the Cox-PH regression coefficients, the sample risk score prediction model was constructed as follows: Risk score $=(-0.605) \mathrm{x}$ expression$\mathrm{ANKSIB}+(-0.179) \mathrm{x}$ expression ${ }_{\mathrm{CAMK} 1}+(-0.496)$ expression $_{\mathrm{CES} 1}+$ $(-1.468) \mathrm{x} \operatorname{expression}_{\mathrm{CTLA} 4}+(0.948) \mathrm{x}$ expression $\mathrm{CXCL}_{2}+(0.379) \mathrm{x}$ expression $_{\text {DNAJC12 }} \times(-0.619) \mathrm{x}$ expression ${ }_{\mathrm{EFNAS}}+(0.317) \mathrm{x}$ expres$\operatorname{sion}_{\mathrm{FEZ1}}+(-0.114) \mathrm{x}$ expression ${ }_{\mathrm{HOPX}}+(-0.039) \mathrm{x}$ expression $_{\mathrm{HPCAL}}$ $+(0.187) \mathrm{x}$ expression ${ }_{\mathrm{IL} 17 \mathrm{RB}}+(-0.348) \mathrm{x}$ expression ${ }_{\mathrm{MUCl}}$.

Based on this model, the risk score of each sample in GSE32918 (the training set) was calculated and the samples were divided into high risk score and low risk score groups according to the median risk score. Kaplan-Meier survival curves were used to evaluate overall survival prognosis in the 167 DLBCL samples in the training set. As shown in Fig. 4A, the patients with a high risk score had significantly shorter survival times $\left(\mathrm{P}=1.84 \times 10^{-8}\right)$. The prediction model had a high sensitivity, with the area under the receiver operating characteristic curve (AUC) of 0.942 . Additionally, the prediction performance of the model was confirmed using the validation set. Patients with high risk scores had shorter survival times $\left(\mathrm{P}=3.96 \times 10^{-2}\right.$; $\mathrm{AUC}=0.889$; Fig. $\left.4 \mathrm{~B}\right)$.

In order to analyze the performance of the prediction model in DLBCL and R-CHOP-treated DLBCL samples,
A
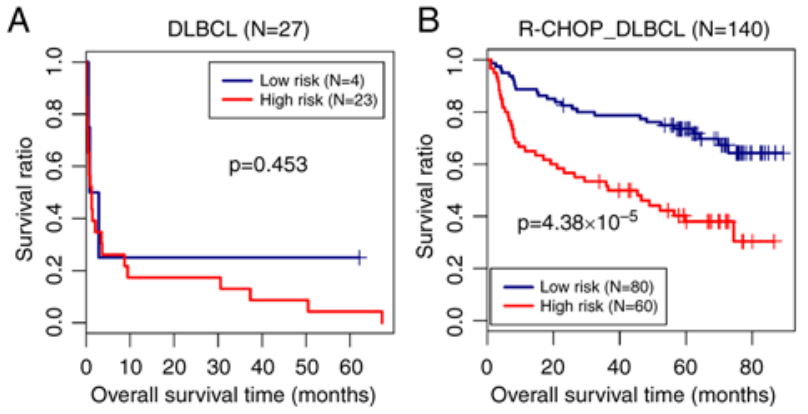

Figure 5. Prediction model and the overall survival Kaplan-Meier curves. Overall survival Kaplan-Meier curves based on the optimal gene combination in (A) DLBCL and (B) R-CHOP treated DLBCL samples. Blue and red curves represent the low risk score and high risk score groups, respectively. DLBCL, diffuse large B-cell lymphoma; R-CHOP, rituximab plus cyclophosphamide, doxorubicin, vincristine, and prednisone immunochemotherapy.

the samples in the training set were divided into DLBCL and R-CHOP-treated DLBCL groups, and the risk score model was analyzed in the two sets of samples. As shown in Fig. 5, the predictive ability of the risk prediction model in the R-CHOP-treated DLBCL group $\left(\mathrm{P}=4.38 \times 10^{-5}\right)$ was superior to that in the untreated DLBCL group $(\mathrm{P}=0.453)$. This indicated that the risk score model had a high prediction power in R-CHOP-treated DLBCLs.

Function annotation of risk associated genes. A total of 729 DEGs were identified comparing the high and low risk score samples in the training set, among which 314 DEGs were significantly downregulated and 415 were significantly upregulated in the high risk score group (Fig. 6A). The volcano plot is shown in Fig. 6A and the heatmap based on the expression level changes of the DEGs, with the risk score, is shown in Fig. 6B. These DEGs were used for functional annotation; 30 significant GO terms (16 $\mathrm{BP}$, nine $\mathrm{CC}$ and five MF) and nine KEGG pathways were identified (Table III and Fig. 7). The DEGs were significantly enriched in functions associated with 'response to DNA damage stimulus', 'cell cycle' and the 'cytokine-cytokine receptor interaction' pathways.

\section{Discussion}

In the present study, 801 DEGs were identified between R-CHOP-treated DLBCL and primary DLBCL samples. From the 801 DEGs, 116 prognosis-associated genes were identified; these genes were significantly enriched in $18 \mathrm{GO}$ terms and eight pathways. Using the Cox-PH model, an optimal combination of 12 genes was selected, and the sample risk score prediction model was constructed and validated. The samples in the training set were divided into high and low risk score groups based on the risk score. The DEGs between high and low risk score groups were significantly enriched in functions associated with 'response to DNA damage stimulus', 'cell cycle' and the 'cytokine-cytokine receptor interaction' pathway.

It has been reported that the signal transduction system, comprising growth factors, cytoplasmic secondary messengers and transmembrane receptor proteins, optimizes the growth and metastasis of tumor cells in malignancies, and is therefore 
A

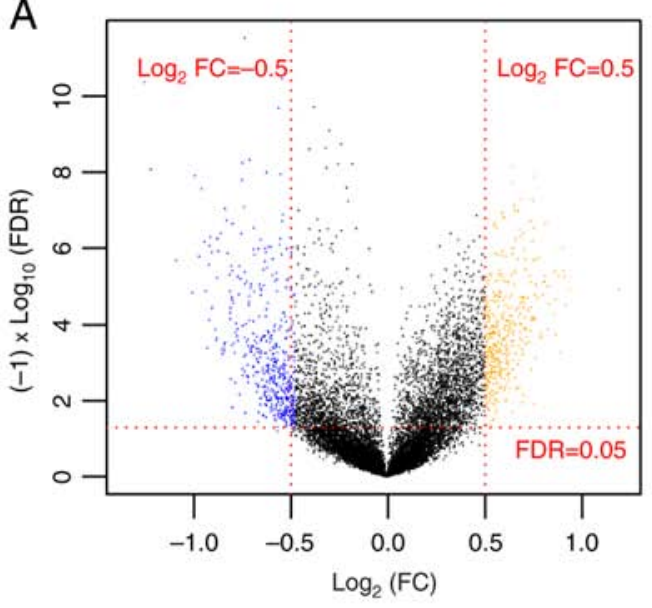

B

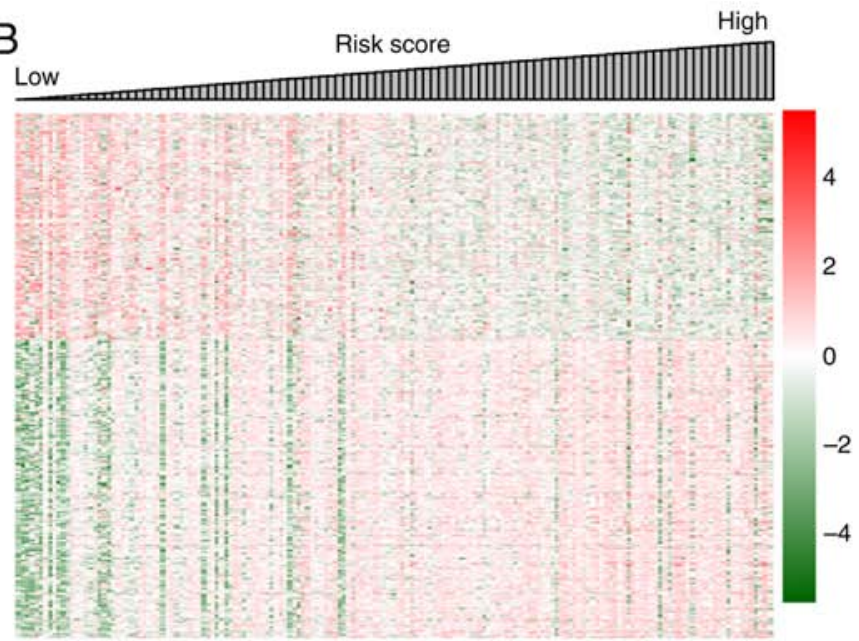

Figure 6. DEGs between high and low risk groups. (A) Volcano plot. Orange and blue dots indicate significantly upregulated and downregulated DEGs, respectively. The red dotted horizontal line indicates an FDR $<0.05$ and the two red dotted vertical lines indicate $\mid l o g F C l>0.5$. (B) Heatmap based on the expression level of DEGs. DEGs, differentially expressed genes; FDR, false discovery rate; FC, fold change.

considered as a target for cancer therapy (27). Functional enrichment analysis showed that calcium/calmodulin dependent protein kinase I (CAMK1), a component of the calmodulin-dependent protein kinase cascade, and hippocalcin like 4 (HPCAL4) were enriched in functions associated with 'signal transduction' (GO:0007165). The roles of CAMKI and HPCAL4 in cancer have not been fully analyzed to the best of our knowledge, but it is speculated that $C A M K 1$ and HPCAL4 may be targets of R-CHOP in DLBCL given their association with signal transduction.

Among the 12 genes, five were found to be associated with 'protein binding' (GO:0005515), including CAMK1, cytotoxic T-lymphocyte associated protein 4 (CTLA4), fasciculation and elongation protein $\zeta 1$ ( FEZ1), HOP homeobox (HOPX), and mucin 16 , cell surface associated (MUC16). CTLA4 is a coinhibitory receptor that regulates T-cell activation during the initiation and maintenance of adaptive immune responses. The blocking of CTLA4 promotes antitumor T-cell immunity (28). In addition, rituximab could enhance the production of CTLA4 by regulatory T-cells (29) and B-cells (30). FEZ1 encodes a $67 \mathrm{kDa}$ leucine-zipper protein with a region similar to cAMP-dependent activated protein (31), which has been found to be mutated in solid tumors (32). In FEZ1 null cancer cells, FEZ1 introduction has been reported to reduce cell growth, while FEZ1 inhibition stimulates cell growth, suggesting a role for FEZ1 in human cancer (33). HOPX belongs to the homeobox gene family and is ubiquitously expressed in normal tissues (34). It has been reported that enforced $H O P X$ expression inhibits tumor progression and that $H O P X$ knockdown restores tumor aggressiveness (35). MUC16 is a member of the mucin family that is reported to be involved in tumorigenicity and therapeutic resistance in pancreatic cancer (36). Moreover, MUC16 is overexpressed in multiple types of cancer and has an important role in acquired resistance to therapy (37). However, to the best of our knowledge, there is no previous evidence showing the association of these DEGs with CD20 or DLBCL. Given the roles of the five genes in cancer, it is speculated that R-CHOP may target CAMK1, CTLA4, FEZ1, HOPX and MUC16. The negative effect of CTLA4 on risk scores in DLBCL samples showed that the increased expression of CTLA4 contributed to the efficiency of rituximab therapy. Similar hypotheses could be made for the $C A M K 1, M U C 16$ and $H O P X$ genes.

The other two DEGs, C-X-C motif chemokine ligand 2 (CXCL2) and ephrin A5 (EFNA5) were found to be involved in several signaling pathways, including the 'Ras signaling pathway', 'chemokine signaling pathway' and 'PI3K-Akt signaling pathway'. These signaling mechanisms are fundamental pathways in normal and tumor cells, even if tumor cells may be more reliant on these pathways (38). The Ras signaling pathway is commonly activated in tumors (38). Rational therapies targeting the Ras signaling pathways inhibit tumor survival, growth and spread (39). The PI3K-Akt signaling pathway regulates multiple tumorigenesis-associated cellular processes, including cell proliferation, growth and motility (40). Mutations or altered expression levels of components of the PI3K-Akt pathway are implicated in human cancer (41). Therefore, several drugs, alone and in combination, including rituximab, targeting the PI3K-Akt signaling pathway are in clinical trials, in both hematologic malignancies and solid tumors $(42,43)$. It has been previously reported that rituximab-induced apoptosis in the B-cell lymphoma cell line SD07 is mediated by PI3K-Akt dephosphorylation or suppression (43). The normal development of cells depends on chemokines and their receptors, and tumor progression also requires stimulation by chemokines (44). CXCL2, which is part of the CXC chemokine family, promotes inflammation and supports tumor growth (45). Taken together, it is speculated that CXCL2 and EFNA5 may be targeted by R-CHOP via these signaling pathways. However, to the best of our knowledge, there is no evidence showing the association of CXCL2 alteration with DLBCL, R-CHOP therapy or rituximab.

Interleukin 17 receptor B $(I L 17 R B)$ was enriched in the 'cytokine-cytokine receptor interaction' pathway (hsa04060). IL17RB, a proinflammatory cytokine, is the receptor of IL17B (46). Cytokines play important roles in the localization of normal or malignant B-cells in tissues (47). IL17RB 
Table III. GO and KEGG pathway enrichment results for DEGs.

A, Biological process

\begin{tabular}{lll}
\hline Term & Count & P-value \\
\hline GO:0006974 response to DNA damage stimulus & 32 & $2.47 \times 10^{-04}$ \\
GO:0006281 DNA repair & 26 & $4.14 \times 10^{-04}$ \\
GO:0045184 establishment of protein localization & 50 & 0.002456 \\
GO:0006259 DNA metabolic process & 36 & 0.002753 \\
GO:0015031 protein transport & 49 & 0.003342 \\
GO:0008104 protein localization & 55 & 0.003533 \\
GO:0022402 cell cycle process & 38 & 0.00521 \\
GO:0033554 cellular response to stress & 38 & 0.005394 \\
GO:0007049 cell cycle & 48 & 0.007516 \\
GO:0051301 cell division & 22 & 0.013451 \\
GO:0022403 cell cycle phase & 28 & 0.016532 \\
GO:0000278 mitotic cell cycle & 25 & 0.024163 \\
GO:0032989 cellular component morphogenesis & 26 & 0.029787 \\
GO:0042127 regulation of cell proliferation & 45 & 0.032887 \\
GO:0000279 M phase & 22 & 0.038695 \\
GO:0042981 regulation of apoptosis & 45 & 0.045193 \\
\hline
\end{tabular}

B, Cellular component

\begin{tabular}{lrr}
\hline Term & Count & P-value \\
\hline GO:0031974 membrane-enclosed lumen & 101 & $5.31 \times 10^{-04}$ \\
GO:0043233 organelle lumen & 99 & $6.35 \times 10^{-04}$ \\
GO:0070013 intracellular organelle lumen & 97 & $6.75 \times 10^{-04}$ \\
GO:0031981 nuclear lumen & 76 & 0.008073 \\
GO:0019898 extrinsic to membrane & 31 & 0.012421 \\
GO:0000793 condensed chromosome & 12 & 0.012999 \\
GO:0009897 external side of plasma membrane & 14 & 0.017106 \\
GO:0005578 proteinaceous extracellular matrix & 21 & 0.028573 \\
GO:0043235 receptor complex & 10 & 0.039703 \\
\hline
\end{tabular}

C, Molecular function

\begin{tabular}{lcr}
\hline Term & Count & P-value \\
\hline GO:0019955 cytokine binding & 11 & 0.014221 \\
GO:0019900 kinase binding & 15 & 0.015941 \\
GO:0005524 ATP binding & 75 & 0.041085 \\
GO:0019899 enzyme binding & 31 & 0.042744 \\
GO:0030554 adenyl nucleotide binding & 79 & 0.046193 \\
\hline
\end{tabular}

D, KEGG pathway

\begin{tabular}{lrr}
\hline Term & Count & P-value \\
\hline hsa04060:Cytokine-cytokine receptor interaction & 20 & 0.002507 \\
hsa04115:p53 signaling pathway & 7 & 0.008288 \\
hsa00590:Arachidonic acid metabolism & 5 & 0.024164 \\
hsa00010:Glycolysis/Gluconeogenesis & 5 & 0.028166 \\
hsa04062:Chemokine signaling pathway & 11 & 0.032858 \\
hsa04660:T cell receptor signaling pathway & 7 & 0.035535
\end{tabular}


Table III. Continued.

D, KEGG pathway

Term

Count

P-value

hsa04514:Cell adhesion molecules (CAMs)

8

0.038001

hsa04110:Cell cycle

7

0.04925

GO, Gene Ontology; KEGG, Kyoto Encyclopedia of Genes and Genomes; DEG, differentially expressed gene.

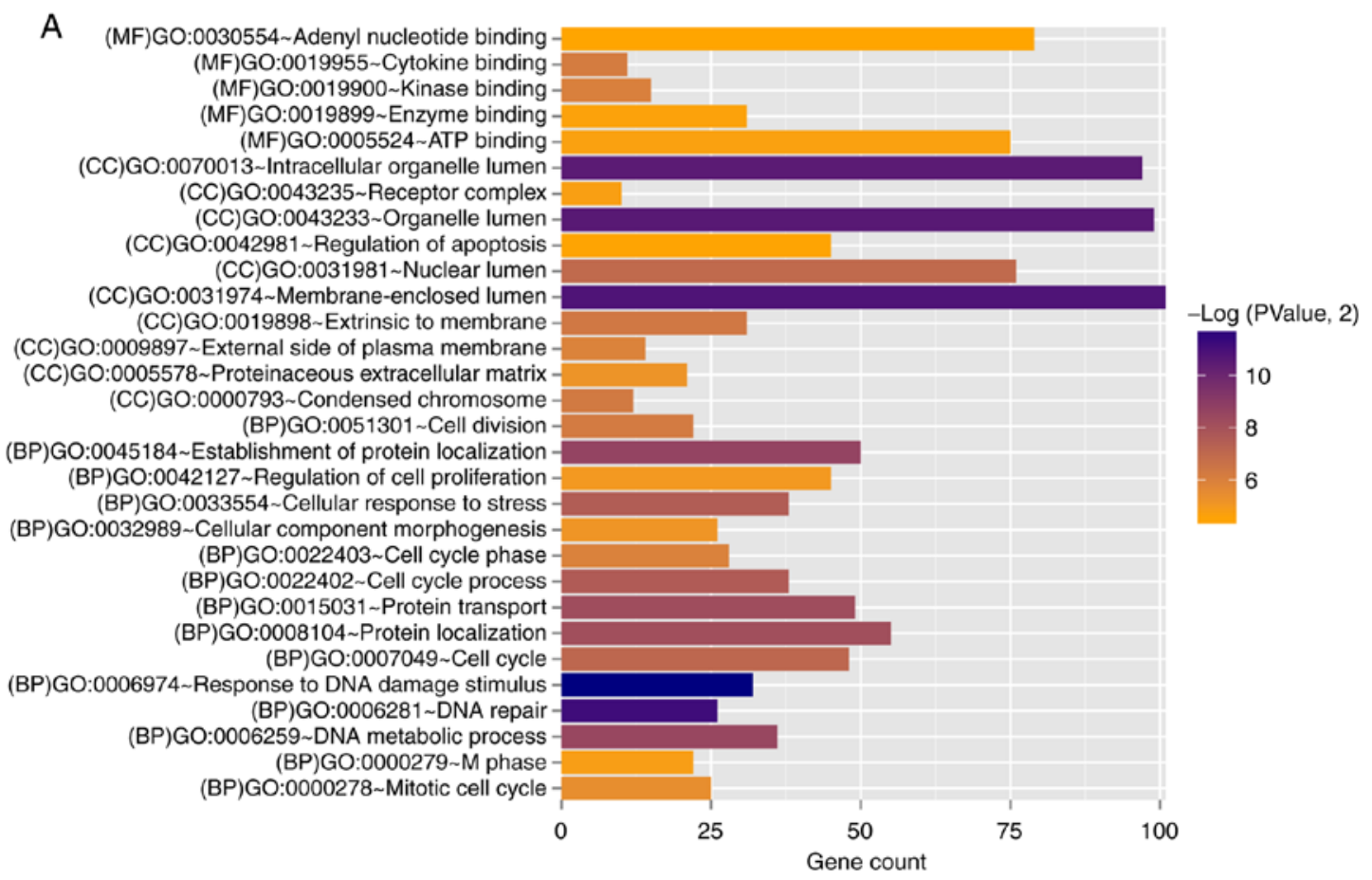

B

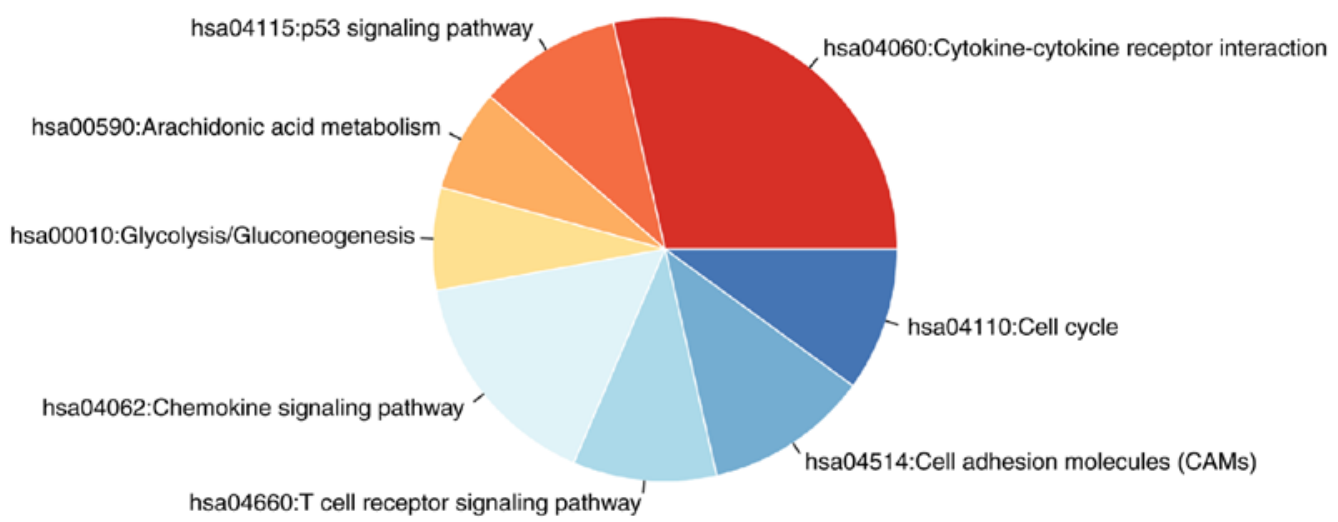

Figure 7. Gene enrichment analysis. (A) Bar graph of the GO terms significantly associated with the differentially expressed genes. The horizontal axis represents the number of genes and the vertical axis shows the term. The color of the bars indicates the significance level. (B) Pie chart of KEGG pathways identified. Each segment represents a different KEGG pathway. The colors represent significant $\mathrm{P}$-values $(\mathrm{P}<0.05)$ for each section; red low $\mathrm{P}$-value, blue high P-value. KEGG, Kyoto Encyclopedia of Genes and Genomes; MF, molecular function; GO, Gene Ontology; CC, cellular component; BP, biological process.

is expressed in endocrine tissues and is expressed in normal mammary epithelial cells at negligible levels (48). However, IL17RB was previously found to be highly expressed in malignant mammary epithelial cells (49). The overexpression of IL17B has been reported to be associated with poor prognosis in breast cancer (50). Previous studies have reported that certain cytokines, including interleukin- 4 and tumor necrosis factor- $\alpha$ (TNF- $\alpha$ ) upregulate the expression of the CD20 antigen, increasing the efficacy of rituximab $(51,52)$. IL-17 and TNF- $\alpha$ are inflammatory cytokines that are co-expressed by $\mathrm{T}$ helper 17 cells in a number of tumors (53). The findings indicate that IL17RB has a role in R-CHOP-mediated therapy for DLBCL. 
In addition to the aforementioned genes, the remaining three DEGs were not enriched in GO terms or pathways. Ankyrin repeat and sterile $\alpha$ motif domain-containing protein $1 \mathrm{~B}(A N K S 1 B)$ is involved in tyrosine kinase signal transduction (54), and is primarily expressed in the brain and testis (55). $A N K S 1 B$ is involved in apoptosis, and thus, has potential functions in cancer development (56). A previous study demonstrated that the expression of $A N K S 1 B$ gene is associated with the development of smoking-related clear cell renal cell carcinoma (57). Carboxylesterase 1 (CES1) is a member of the carboxylesterase family and is important in phase I metabolism (58). Carboxylesterases mediate the metabolism of drug compounds with thioester, ester and amide linkages, and the detoxification of xenobiotics (59). Carboxylesterases are found to hydrolyze two anticancer agents, capecitabine and irinotecan (60). DnaJ heat shock protein family member $\mathrm{C} 12$ (DNAJC12) encodes a member of a subclass of the heat shock protein $40 \mathrm{kDa}$ family that is involved in a number of important biological functions $(61,62)$. High expression of DNAJC12 functions as a negative predictive factor for the response to neoadjuvant concurrent chemotherapy in rectal cancer (63). Little information is available regarding these genes, and their role in DLBCL and other diseases. The combination of these genes in the risk predication model suggests their important roles in DLBCL therapy. It is speculated that $A N K S 1 B, C E S 1$ and DNAJC12 may serve as important target genes in R-CHOP therapy in DLBCL.

In conclusion, the optimal combination of 12 genes to predict prognosis risk, including CAMK1, HPCAL4, CXCL2 and EFNA5, was selected based on the differential expression of these genes between R-CHOP-treated DLBCL and primary DLBCL groups. These genes were utilized in the construction of the prognosis prediction model in DLBCL after R-CHOP treatment. These genes may also serve as target genes of R-CHOP in DLBCL. To the best of our knowledge, most of these DEGs have not been reported to be associated with DLBCL and CD20 or rituximab-mediated therapy, highlighting the novel insights the present study provides into the pathogenesis and treatment of DLBCL.

\section{Acknowledgements}

Not applicable.

\section{Funding}

The present study was supported by the National Natural Science Foundation of China (grant no. 81600161).

\section{Availability of data and materials}

The datasets used and/or analyzed during the current study are available from the corresponding author on reasonable request.

\section{Authors' contributions}

$\mathrm{RL}$ and $\mathrm{ZC}$ were responsible for the conception and design of the research and the manuscript drafting. YG performed the revision for important intellectual content. GZ, YG, SW,
$\mathrm{QH}$ and $\mathrm{BC}$ were responsible for the acquisition, analysis and interpretation of data. $\mathrm{QH}$ and $\mathrm{BC}$ were involved in the manuscript revision. All authors approved the final revision.

\section{Ethics approval and consent to participate}

Not applicable.

\section{Patient consent for publication}

Not applicable.

\section{Competing interests}

The authors declare that they have no competing interests.

\section{References}

1. Sabattini E, Bacci F, Sagramoso C and Pileri SA: WHO classification of tumours of haematopoietic and lymphoid tissues in 2008: An overview. Pathologica 102: 83-87, 2010.

2. Dreyling M, Thieblemont C, Gallamini A, Arcaini L, Campo E, Hermine O, Kluin-Nelemans JC, Ladetto M, Le Gouill S, Iannitto E, et al: ESMO Consensus conferences: Guidelines on malignant lymphoma. part 2: Marginal zone lymphoma, mantle cell lymphoma, peripheral T-cell lymphoma. Ann Oncol 24: 857-877, 2013.

3. Ganjoo KN, An CS, Robertson MJ, Gordon LI, Sen JA, Weisenbach J, Li S, Weller EA, Orazi A and Horning SJ: Rituximab, bevacizumab and CHOP (RA-CHOP) in untreated diffuse large B-cell lymphoma: Safety, biomarker and pharmacokinetic analysis. Leuk Lymphoma 47: 998-1005, 2006.

4. Patil Y, Sadhukha T, Ma L and Panyam J: Nanoparticle-mediated simultaneous and targeted delivery of paclitaxel and tariquidar overcomes tumor drug resistance. J Control Release 136: 21-29, 2009.

5. Cultrera JL and Dalia SM: Diffuse large B-cell lymphoma: Current strategies and future directions. Cancer Control 19: 204-213, 2012.

6. Coiffier B, Lepage E, Briere J, Herbrecht R, Tilly H, Bouabdallah R, Morel P, Van Den Neste E, Salles G, Gaulard $\mathrm{P}$, et al: $\mathrm{CHOP}$ chemotherapy plus rituximab compared with CHOP alone in elderly patients with diffuse large-B-cell lymphoma. N Engl J Med 346: 235, 2002.

7. Maloney DG, Grillo-López AJ, White CA, Bodkin D, Schilder RJ, Neidhart JA, Janakiraman N, Foon KA, Liles TM, Dallaire BK, et al: IDEC-C2B8 (Rituximab) anti-CD20 monoclonal antibody therapy in patients with relapsed low-grade non-Hodgkin's lymphoma. Blood 90: 2188-2195, 1997.

8. Mclaughlin P, Grillo-López AJ, Link BK, Levy R, Czuczman MS, Williams ME, Heyman MR, Bence-Bruckler I, White CA, Cabanillas F, et al: Rituximab chimeric anti-CD20 monoclonal antibody therapy for relapsed indolent lymphoma: Half of patients respond to a four-dose treatment program. J Clin Oncol 16: 2825-2833, 1998.

9. Habermann TM, Weller EA, Morrison VA, Gascoyne RD, Cassileth PA, Cohn JB, Dakhil SR, Woda B, Fisher RI, Peterson BA and Horning SJ: Rituximab-CHOP versus CHOP alone or with maintenance rituximab in older patients with diffuse large B-cell lymphoma. J Clin Oncol 24: 3121-3127, 2006.

10. National Comprehensive Cancer Network: NCCN Clinical Practice Guidelines in Oncology. 2003.

11. Pettengell R, Bosly A, Szucs TD, Jackisch C, Leonard R, Paridaens R, Constenla $\mathrm{M}$ and Schwenkglenks M; Impact of Neutropenia in Chemotherapy-European Study Group (INC-EU): Multivariate analysis of febrile neutropenia occurrence in patients with non-Hodgkin lymphoma: Data from the INC-EU Prospective Observational European Neutropenia Study. Br J Haematol 144: 677-685, 2009.

12. Sehn LH, Scott DW, Chhanabhai M, Berry B, Ruskova A, Berkahn L, Connors JM and Gascoyne RD: Impact of concordant and discordant bone marrow involvement on outcome in diffuse large B-cell lymphoma treated with R-CHOP. J Clin Oncol 29: 1452-1457, 2011. 
13. Barrans SL, Crouch S, Care MA, Worrillow L, Smith A, Patmore R, Westhead DR, Tooze R, Roman E and Jack AS: Whole genome expression profiling based on paraffin embedded tissue can be used to classify diffuse large B-cell lymphoma and predict clinical outcome. Br J Haematol 159: 441-453, 2012.

14. Scholtysik R, Kreuz M, Hummel M, Rosolowski M, Szczepanowski M, Klapper W, Loeffler M, Trümper L, Siebert R and Küppers R; Molecular Mechanisms in Malignant Lymphomas Network Project of the Deutsche Krebshilfe: Characterization of genomic imbalances in diffuse large B-cell lymphoma by detailed SNP-chip analysis. Int J Cancer 136: 1033-1042, 2015.

15. Ritchie ME, Phipson B, Wu D, Hu Y, Law CW, Shi W and Smyth GK: limma powers differential expression analyses for RNA-sequencing and microarray studies. Nucleic Acids Res 43: e47, 2015.

16. Li S, Sun YN, Zhou YT, Zhang CL, Lu F, Liu J and Shang XM: Screening and identification of microRNA involved in unstable angina using gene-chip analysis. Exp Ther Med 12: 2716-2722, 2016.

17. Parrish RS and Spencer HJ III: Effect of normalization on significance testing for oligonucleotide microarrays. J Biopharm Stat 14: 575-589, 2004.

18. Kaminskyi R, Kunanets N, Pasichnyk V, Rzheuskyi A and Khudyi A: Recovery gaps in experimental data. In: COLINS, pp. 108-118, 2018

19. Bolstad BM, Irizarry RA, Åstrand M and Speed TP: A comparison of normalization methods for high density oligonucleotide array data based on variance and bias. Bioinformatics 19: 185-193, 2003.

20. Szekely GJ and Rizzo ML: Hierarchical clustering via joint between-within distances: Extending Ward's minimum variance method. J Classification 22: 151-183, 2005.

21. Press W, Teukolsky S, Vetterling W and Flannery B: Section 16.4. Hierarchical clustering by phylogenetic trees. Numerical recipes: The art of scientific computing: 868-881, 2007.

22. Wang L, Cao C, Ma Q, Zeng Q, Wang H, Cheng Z, Zhu G, Qi J, Ma H, Nian $\mathrm{H}$ and Wang Y: RNA-seq analyses of multiple meristems of soybean: Novel and alternative transcripts, evolutionary and functional implications. BMC Plant Biol 14: 169, 2014

23. Wang P, Wang Y, Hang B, Zou X and Mao JH: A novel gene expression-based prognostic scoring system to predict survival in gastric cancer. Oncotarget 7: 55343-55351, 2016.

24. Huang da W, Sherman BT and Lempicki RA: Systematic and integrative analysis of large gene lists using DAVID bioinformatics resources. Nat Protoc 4: 44-57, 2009.

25. Tibshirani R: THE lasso method for variable selection in the COX model. Stat Med 16: 385-395, 1997.

26. Goeman JJ: L1 penalized estimation in the Cox proportional hazards model. Biom J 52: 70-84, 2010.

27. Adjei AA and Hidalgo M: Intracellular signal transduction pathway proteins as targets for cancer therapy. J Clin Oncol 23 5386-5403, 2005.

28. Wang D, Zhang B, Gao H, Ding G, Wu Q, Zhang J, Liao L and Chen $\mathrm{H}$ : Clinical research of genetically modified dendritic cells in combination with cytokine-induced killer cell treatment in advanced renal cancer. BMC Cancer 14: 251, 2014

29. Sinha A and Bagga A: Rituximab therapy in nephrotic syndrome: Implications for patients' management. Nat Rev Nephrol 9: 154-169, 2013.

30. Sfikakis P, Souliotis V, Fragiadaki K, Moutsopoulos H, Boletis $\mathbf{J}$ and Theofilopoulos A: Increased expression of the Foxp3 functional marker of regulatory $t$ cells following $b$ cell depletion with rituximab in patients with lupus nephritis. Clin Immunol 123: 66-73, 2007.

31. Ishii H, Baffa R, Numata SI, Murakumo Y, Rattan S, Inoue H, Mori M, Fidanza V, Alder H and Croce CM: The FEZ1 gene at chromosome 8p22 encodes a leucine-zipper protein, and its expression is altered in multiple human tumors. Proc Natl Acad Sci USA 96: 3928-3933, 1999.

32. Vecchione A, Ishii H, Shiao YH, Trapasso F, Rugge M, Tamburrino JF, Murakumo Y, Alder H, Croce CM and Baffa R: Fez1/lzts1 alterations in gastric carcinoma. Clin Cancer Res 7: 1546-1552, 2001

33. Cabeza-Arvelaiz Y, Sepulveda JL, Lebovitz RM, Thompson TC and Chinault AC: Functional identification of LZTS1 as a candidate prostate tumor suppressor gene on human chromosome 8p22. Oncogene 20: 4169-4179, 2001.

34. Kikuchi M, Katoh $\mathrm{H}$, Waraya M, Tanaka $\mathrm{Y}$, Ishii S, Tanaka T, Nishizawa N, Yokoi K, Minatani N, Ema A, et al: Epigenetic silencing of HOPX contributes to cancer aggressiveness in breast cancer. Cancer Lett 384: 70-78, 2017.
35. Cheung WK, Zhao M, Liu Z, Stevens LE, Cao PD, Fang JE, Westbrook TF and Nguyen DX: Control of alveolar differentiation by the lineage transcription factors GATA6 and HOPX inhibits lung adenocarcinoma metastasis. Cancer Cell 23 725-738, 2013.

36. Shukla SK, Gunda V, Abrego J, Haridas D, Mishra A, Souchek J, Chaika NV, Yu F, Sasson AR, Lazenby AJ, et al: MUC16-mediated activation of mTOR and c-MYC reprograms pancreatic cancer metabolism. Oncotarget 6: 19118-19131, 2015.

37. Aithal A, Rauth S, Kshirsagar P, Shah A, Lakshmanan I, Junker WM, Jain M, Ponnusamy MP and Batra SK: MUC16 as a novel target for cancer therapy. Expert Opin Ther Targets 22: 675-686, 2018

38. Downward J: Targeting RAS signalling pathways in cancer therapy. Nat Rev Cancer 3: 11-22, 2003.

39. Slack C, Alic N, Foley A, Cabecinha M, Hoddinott MP and Partridge L: The Ras-Erk-ETS-signaling pathway is a drug target for longevity. Cell 162: 72-83, 2015.

40. Huang Y, Zhang J, Hou L, Wang G, Liu H, Zhang R, Chen X and Zhu J: LncRNA AK023391 promotes tumorigenesis and invasion of gastric cancer through activation of the PI3K/Akt signaling pathway. J Exp Clin Cancer Res 36: 194, 2017.

41. Martini M, De Santis M, Braccini L, Gulluni F and Hirsch E: PI3K/AKT signaling pathway and cancer: An updated review. Ann Med 46: 372-383, 2014.

42. Mayer IA and Arteaga CL: The PI3K/AKT pathway as a target for cancer treatment. Annu Rev Med 67: 11-28, 2015.

43. Nakamaki T, Baba Y, Abe M, Murai S, Watanuki M, Kabasawa N, Yanagisawa K, Hattori N, Kawaguchi Y, Arai N, et al: Rituximab-induced CD20-mediated signals and suppression of PI3K-AKT pathway cooperates to inhibit B-cell lymphoma growth by down-regulation of Myc. Blood 128: 5312,2016

44. Nagasawa T, Hirota S, Tachibana K, Takakura N, Nishikawa S, Kitamura Y, Yoshida N, Kikutani H and Kishimoto T: Defects of B-cell lymphopoiesis and bone-marrow myelopoiesis in mice lacking the CXC chemokine PBSF/SDF-1. Nature 382: 635-638, 1996.

45. Zhu Q, Peng J, Qin $\mathrm{H}$ and $\mathrm{Yu} \mathrm{W}$ : The role of CXC chemokines and their receptors in the progression and treatment of tumors. J Mol Histol 43: 699-713, 2012.

46. Hunninghake GM, Chu JH, Sharma SS, Cho MH, Himes BE, Rogers AJ, Murphy A, Carey VJ and Raby BA: The CD4+ T-cell transcriptome and serum IgE in asthma: IL17RB and the role of sex. BMC Pulm Med 11: 17, 2011.

47. Husson H, Carideo EG, Cardoso AA, Lugli SM, Neuberg D, Munoz O, de Leval L, Schultze J and Freedman AS: MCP-1 modulates chemotaxis by follicular lymphoma cells. $\mathrm{Br}$ J Haematol 115: 554-562, 2001.

48. Alinejad V, Dolati S, Motallebnezhad M and Yousefi M: The role of IL17B-IL17RB signaling pathway in breast cancer. Biomed Pharmacother 88: 795-803, 2017.

49. Sharma S, Rajaram S, Sharma T, Goel N, Agarwal S and Banerjee BD: Role of BRCA1 and BRCA2 gene mutations in epithelial ovarian cancer in Indian population: A pilot study. Int J Biochem Mol Biol 5: 1-10, 2014.

50. Furuta S, Jeng YM, Zhou L, Huang L, Kuhn I, Bissell MJ and Lee WH: IL-25 causes apoptosis of IL-25R-expressing breast cancer cells without toxicity to nonmalignant cells. Sci Transl Med 3: 78ra31, 2011

51. Pievani A, Belussi C, Klein C, Rambaldi A, Golay J and Introna M: Enhanced killing of human B-cell lymphoma targets by combined use of cytokine-induced killer cell (CIK) cultures and anti-CD20 antibodies. Blood 117: 510-518, 2011.

52. Venugopal P, Sivaraman S, Huang X, Nayini J, Gregory S and Preisler H: Effects of cytokines on CD20 antigen expression on tumor cells from patients with chronic lymphocytic leukemia. Leuk Res 24: 411-415, 2000.

53. Wang X, Yang L, Huang F, Zhang Q, Liu S, Ma L and You Z: Inflammatory cytokines IL-17 and TNF- $\alpha$ up-regulate PD-L1 expression in human prostate and colon cancer cells. Immunol Lett 184: 7-14, 2017.

54. Herberich SE, Klose R, Moll I, Yang WJ, Wüstehube-Lausch J and Fischer A: ANKS1B interacts with the cerebral cavernous malformation protein-1 and controls endothelial permeability but not sprouting angiogenesis. PLoS One 10: e0145304, 2015.

55. Fu X, McGrath S, Pasillas M, Nakazawa S and Kamps MP: EB-1, a tyrosine kinase signal transduction gene, is transcriptionally activated in the $t(1 ; 19)$ subset of pre-B ALL, which express oncoprotein E2a-Pbx1. Oncogene 18: 4920-4929, 1999. 
56. Evan GI and Vousden KH: Proliferation, cell cycle and apoptosis in cancer. Nature 411: 342-348, 2001.

57. Eckel-Passow JE, Serie DJ, Bot BM, Joseph RW, Cheville JC and Parker AS: ANKS1B is a smoking-related molecular alteration in clear cell renal cell carcinoma. BMC Urol 14: 14, 2014.

58. Satoh T and Hosokawa M: Structure, function and regulation of carboxylesterases. Chem Biol Interact 162: 195-211, 2006.

59. Redinbo MR and Potter PM: Mammalian carboxylesterases: From drug targets to protein therapeutics. Drug Discov Today 10: 313-325, 2005.

60. Pratt SE, Durland-Busbice S, Shepard RL, Heinz-Taheny K, Iversen PW and Dantzig AH: Human carboxylesterase-2 hydrolyzes the prodrug of gemcitabine (LY2334737) and confers prodrug sensitivity to cancer cells. Clin Cancer Res 19: 1159-1168, 2013.

61. Anikster Y, Haack TB, Vilboux T, Pode-Shakked B, Thöny B Shen N, Guarani V, Meissner T, Mayatepek E, Trefz FK, et al: Biallelic mutations in DNAJC12 cause hyperphenylalaninemia, dystonia, and intellectual disability. Am J Hum Genet 100: 257-266, 2017
62. Jung-KC K, Himmelreich N, Prestegård KS, Shi TS, Scherer T, Ying M, Jorge-Finnigan A, Thöny B, Blau N and Martinez A: Phenylalanine hydroxylase variants interact with the co-chaperone DNAJC12. Hum Mutat 40: 483-494, 2019.

63. He HL, Lee YE, Chen HP, Hsing CH, Chang IW, Shiue YL, Lee SW, Hsu CT, Lin LC, Wu TF and Li CF: Overexpression of DNAJC12 predicts poor response to neoadjuvant concurrent chemoradiotherapy in patients with rectal cancer. Exp Mol Pathol 98: 338-345, 2015

This work is licensed under a Creative Commons Attribution-NonCommercial-NoDerivatives 4.0 International (CC BY-NC-ND 4.0) License. 